\title{
Thermomechanical behaviour of thermoelectric SMA actuators
}

\author{
R.V.N. Melnik and A.J. Roberts ${ }^{1}$ \\ CSIRO Mathematical and Information Sciences, Macquarie University Campus, North Ryde, \\ NSW 2113, Australia \\ ${ }^{1}$ Department of Mathematics and Computing, University of Southern Queensland, QLD 4350, \\ Australia
}

\begin{abstract}
In this paper we analyse numerically thermomechanical behaviour of a sandwich-type actuator where a shape memory alloy layer is located between two oppositely doped semiconductors. The mathematical model for this analysis is based on a coupled system of partial differential equations with constitutive equations taken in the Falk form. The system is solved using an efficient differential-algebraic solver and computational results describing thermomechanical fields in such devices are presented.
\end{abstract}

\section{Introduction}

Shape memory alloys (SMAs) are widely used as actuators for shape and vibration control of structures. Since the response time of a SMA can be controlled by a combined effect of heat transfer (to and from the device) and mechanical loading, in order to quantify and ultimately optimise heating/cooling conditions heat transfer models alone are not sufficient. Although many efforts have been concentrated on the development of thermal models for stress-free SMA samples $[6,3]$, the development of full thermoelectromechanical models and numerical methods for their solutions constitutes an important problem in theory and applications of shape memory materials $[2,7,9,11]$.

In order to increase the rates of cooling in high frequency applications different methodologies have been proposed and compared with more traditional techniques such as free and forced convection [1]. Some such methodologies require dealing with multilayered structures which complicates the analysis and modelling of material dynamics [8]. In this paper we consider sandwich-type SMA actuators with semiconductor modules, conceptual design of which has been explained in $[12,6]$. The modelling region for a representative example of this type of devices is given in Fig. 1. The principle of work of such devices is based on the use of thermoelectric effects. Such effects (namely, the Seebeck, Peltier, and Thomson effects) are widely used in thermoelectric circuits to produce useful heating/cooling and/or power generation, and the areas of applications of thermoelectricity-based devices range from refrigeration/generation tools to nuclear industry and solar energy converters. In this paper our choice for the thermoelectric cooling "cover" of the SMA module is due to the fact that as thermoelectric materials semiconductors and semiconductor alloys are often superior to metals. We limit our consideration to semiconductor modules consisting of bismuth telluride $\left(\mathrm{Bi}_{2} \mathrm{Te}_{3}\right)$ n- and p-type thermocouples.

The foundations for modelling thermoelectric devices have been laid by the group of researchers led by Ioffe [5] and since then various models of thermoelectricity have been extensively developed in the context of specific (both steady-state and transient) applications by many researchers (see, for example, $[4,12,1,6]$ and references therein). In the context of sandwich-type SMA actuators consisting of SMA and semiconductor layers, due to the Peltier effect, the "heat pumping" along a temperature gradient leads to the heat absorption at the high temperature 


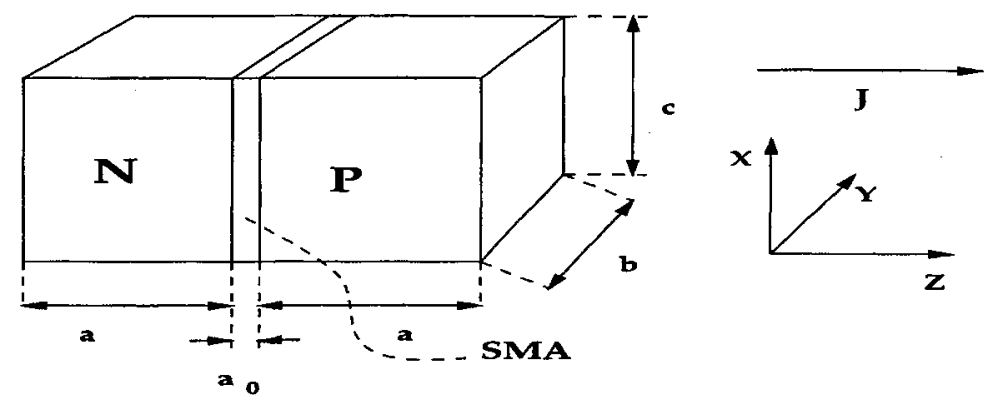

Figure 1: Thermoelectric sandwich-type SMA device with semiconductor layers

junction and the heat dissipation at the cold junction. In particular, as follows from the analysis conducted in [7] if the current is directed from $\mathrm{N}$ to $\mathrm{P}$ semiconductor, the Peltier effect leads the heat loss by the SMA at both interfaces, N-SMA and SMA-P, and these interfaces act as heat sinks for the SMA.

In contrast to the Joule heat the Peltier heat is linear, and since the rate at which thermal energy is transfered from the hot to the cold surface is directly proportional to the carrier current the energy removed from the surface surrounding the SMA layer can be estimated. The procedure for computation of the energy conversion efficiency of such SMA actuators can be found, for example, in [7]. Although the analysis of mathematical models for sandwich-type SMA actuators accounting for the Peltier effect has been a subject of intensive research during recent years $[6,3]$, this analysis is typically limited to the thermal field and, with some noticeable exception [7], little attention has been been paid to the description of coupled thermomechanical fields during phase transformations in these types of devices.

\section{Governing equations and numerical analysis}

Coupled thermomechanical models play an increasingly important role in the design of SMA devices and structures $[2,7,9,11]$. In many applications the analysis of such models and the development of numerical methods for their solution are complicated due to the nonstationary character of the problem and the need to account for the transient heat exchange with the surrounding environment or other layers of the structure. For sandwich-type SMA actuators most studies were devoted to thermoelectric effects under the assumption of uniformity of temperature in the SMA layer [7] without taking into account the coupling between thermal and mechanical fields $[6,3]$.

In this paper we apply a general model investigated in our previous papers $[9,10]$ which consists of the equation of motion and the energy balance equation

$$
\rho \frac{\partial^{2} \mathbf{u}}{\partial t^{2}}=\nabla_{\boldsymbol{x}} \cdot \boldsymbol{\sigma}+\mathbf{f}, \quad \rho \frac{\partial e}{\partial t}=\nabla \cdot \mathbf{q}+\boldsymbol{\sigma}^{T}:(\nabla \mathbf{v})+g
$$

where $\mathbf{u}$ is the vector of spatial displacements, $\boldsymbol{\sigma}$ is the stress tensor, $e$ is the internal energy, $\mathbf{q}$ is the heat flux, $\rho$ is the density of the SMA material, $f$ and $g$ are given functions of mechanical and thermal loadings, respectively. We assume (the Cauchy relation between stress and displacements 
is valid for most SMA applications [7])

$$
\epsilon_{i j}=\frac{1}{2}\left(\frac{\partial u_{i}}{\partial x_{j}}+\frac{\partial u_{j}}{\partial x_{i}}\right), \quad i, j=1,2,3 ; \quad \mathbf{v}=\frac{\partial u}{\partial t} ; \quad \boldsymbol{\sigma}=\frac{\partial \Psi}{\partial \boldsymbol{\epsilon}}
$$

where $\epsilon$ is the symmetric strain tensor, $\mathbf{v}$ is the velocity, and $\Psi$ is the free energy function. If the phase transformation is induced by heating (with or without the presence of body forces), the stress in the SMA layer is produced, and the mechanical field in this case can be adequately described with the nonstationary equation for the conservation of momentum (the equation of motion) as indicated by system (1). A special case of this system has been recently studied in [7] under the assumption of negligible body forces and the temperature gradients in the SMA layer, and the stationarity of the equation for the conservation of momentum.

The presence of thermoelectric semiconductor layers complicates the analysis of the problem and the modelling of thermomechanical fields in the SMA layer. One reason for this is that in this case the thermal energy (heat) flux in the SMA layer with temperature $\theta$ depends on the current density vector $\mathbf{j}=\mathbf{j}(\mathbf{x}, t)$

$$
\nabla \mathbf{q}=\nabla(k \nabla \theta)+\rho|\mathbf{j}|^{2}
$$

where the last term is the Joule term (the function $g$ in (1) models contributions of heat sources, excluding Joule heating) which is negligible during phase transformations. Further, following [7] we assume that the electrical transients are substantially smaller compared to the thermomechanical transients, and as a result of this assumption we reduce the continuity equation for charges to (the current density vector is directed along the z-direction as depicted in Fig. 1)

$$
\nabla \cdot \mathbf{j}=0, \quad \mathbf{j}=J(t) \mathbf{n}_{z}
$$

(computations were performed for $J=-2 \mathrm{~A} / \mathrm{mm}^{2}$ ).

Mechanical boundary conditions at the interfaces of SMA with semiconductor layers were modelled either with "pinned ends" or by given stress for the SMA layer (stress state is uniaxial along the x-direction as depicted in Fig. 1). An approximation for thermal boundary conditions at the interfaces of SMA with semiconductor layers can be derived using the methodology developed in $[6,3]$ where under certain simplifying assumptions (in particular, where $a_{0} / a$ is relatively small) the three-layer problem was reduced to a two layer problem by incorporating the SMA material into the N-P interface (computational results reported below were obtained for $a_{0}=1 \mathrm{~cm}, a=2 \mathrm{~cm}$ ). In the one-dimensional case, the system for approximating temperature in the $\mathrm{P}$ semiconductor layer has the form:

$$
\left\{\begin{array}{l}
C_{v}^{P} \frac{\partial T_{P}}{\partial t}=k_{P} \frac{\partial^{2} T_{P}}{\partial x^{2}}+\rho_{P} J^{2}-h \frac{P}{A}\left(T_{P}-T_{0}\right), \quad a_{0} / 2<x<a_{0}+a \\
T_{P}(x, 0)=T_{0}, \quad T_{P}\left(a_{0} / 2+a, t\right)=T_{0} \\
2 k_{P} \frac{\partial T_{P}}{\partial x}+\rho_{S} a_{0} J^{2}-a_{0} h \frac{P}{A}\left(T_{P}-T_{0}\right)=-2 T_{P} \alpha_{P} J+C_{v} a_{0} \frac{\partial T_{P}}{\partial t}, \quad x=a_{0} / 2,
\end{array}\right.
$$

where $A=b c$ and $P=2(b+c)$ are the area and perimeter of the cross section of the device, $\rho_{S}$ and $\rho_{P}$ are electrical resistivities of the SMA and semiconductor layer, respectively, $h$ is the heat convection coefficient (results reported below were obtained for $b=c=0.5 \mathrm{~cm}$ and $h=2.5 \times 10^{-5} \mathrm{~g} / \mathrm{ms}^{3} \mathrm{~K}$ ). Throughout the text the variable and coefficients with sub/superscripts $N$ and $P$ are reserved for the $\mathrm{N}$ and $\mathrm{P}$ semiconductor layers, respectively. Finally, we note that 
although the Peltier coefficient determines the cooling capacity of a thermoelectric "cooler", it is the Seebeck coefficient (i.e. thermal emf or thermoelectric power coefficient denoted in (5) as $\alpha_{P}$ ) which is most easily measured (the relation between them is given by the first Kelvin relation $[5,4])$.

In order to approximate the dependency between $T_{P}$ and $\frac{\partial T_{P}}{\partial x}$ at $x=a_{0} / 2$ in [3] it was proposed to reduce the interface condition to an "independent" integro-differential equation in the form

$$
\int_{0}^{t} G(t-\tau)\left(\frac{d T_{P}}{d t}(\tau)+\nu_{1} T_{P}(\tau)\right) d \tau+\mu\left(T_{P}\right) \frac{d T_{P}}{d t}(t)+\nu_{2}(t) T_{P}(t)=\mathcal{F}(t)
$$

with given functions $\mu, \nu_{i}, i=1,2, G$ and $\mathcal{F}$. Solving the latter problem will lead to the solution of (5) via standard procedures and having the interface boundary conditions at SMA-P we can solve the system (1) using efficient numerical method described in our previous papers $[9,11]$. The thermomechanical coupling, including temperature regimes for phase transitions, is included in the free energy function, as explained below, and in determining an approximate law of the heat exchange at the boundary interface we assume that in the vicinity of this interface variations in $C_{v}$ can be neglected (the dependency of $C_{v}$ on $J(t)$ can be incorporated via an appropriate interpolation procedure). Assuming that the first equation in (5) is satisfied at the boundary allows us to express $\frac{d T}{d t}\left(a_{0} / 2, t\right)$ from that equation and substitute it into the interface boundary condition. Within the small variation in thermal spatial gradients at the boundary interface in the semiconductor layer this results in the following relationship for the boundary interface condition

$$
2 k_{P} \frac{\partial T_{P}}{\partial x}=\left(T_{P}-T_{0}\right)\left[a_{0} h \frac{P}{A}-a_{0} \frac{C_{v}}{C_{v}^{P}} h \frac{P}{A}\right]-2 T_{P} \alpha_{P} J-J^{2} a_{0}\left[\rho_{S}-\rho_{P} \frac{C_{v}}{C_{v}^{P}}\right] .
$$

This approximation allows us addressing the problem of thermomechanical coupling in the SMA layer. For computation reported below we used the following coefficients and material parameters.

\begin{tabular}{|l|c|c|}
\hline Coefficient & SMA & Semiconductor (P) \\
\hline Seebeck coefficient $\left(\mathrm{cm}^{2} \mathrm{~g} / \mathrm{ms}^{3} \mathrm{AK}\right)$ & $1.2 \times 10^{-7}$ & $2.15 \times 10^{-6}$ \\
Thermal conductivity $\left(\mathrm{cmg} / \mathrm{ms}^{3} \mathrm{~K}\right)$ & $1.9 \times 10^{-2}$ & $1.63 \times 10^{-4}$ \\
Heat capacity $\left(\mathrm{g} /\left(\mathrm{ms}^{3} \mathrm{cmK}\right)\right.$ & $2.12 \times 10^{-2}$ & $4.35 \times 10^{-2}$ \\
Electrical resistivity $\left(\mathrm{cm}^{3} \mathrm{~g} / \mathrm{ms}^{3} A^{2}\right)$ & $6.32 \times 10^{-7}$ & $1.15 \times 10^{-5}$ \\
\hline
\end{tabular}

With approximation (7) we performed a series of computational experiments using the model (1) in the 1D case. The coupling phenomenon was modelled with the middle term in the following expression for the free energy function

$$
\Psi(\theta, \epsilon)=\psi_{0}(\theta)+\psi_{1}(\theta) \psi_{2}(\epsilon)+\psi_{3}(\epsilon)
$$

where specific forms for the thermal $\left(\psi_{0}\right)$, mechanical $\left(\psi_{3}\right)$ and coupled thermomechanical $\left(\psi_{1} \psi_{2}\right)$ contributions were taken in the Falk form as in [9]. Taking into account that $e=\Psi-\theta \frac{\partial \Psi}{\partial \theta}$ the system (1) was reduced to the following form

$$
\left\{\begin{array}{l}
C_{v} \frac{\partial \theta}{\partial t}-k_{1} \theta \frac{\partial u}{\partial x} \frac{\partial^{2} u}{\partial t \partial x}-\frac{\partial}{\partial x}\left(k \frac{\partial \theta}{\partial x}\right)=g \\
\rho \frac{\partial^{2} u}{\partial t^{2}}-\frac{\partial}{\partial x}\left[k_{1} \frac{\partial u}{\partial x}\left(\theta-\theta_{1}\right)-k_{2}\left(\frac{\partial u}{\partial x}\right)^{3}+k_{3}\left(\frac{\partial u}{\partial x}\right)^{5}\right]=f
\end{array}\right.
$$



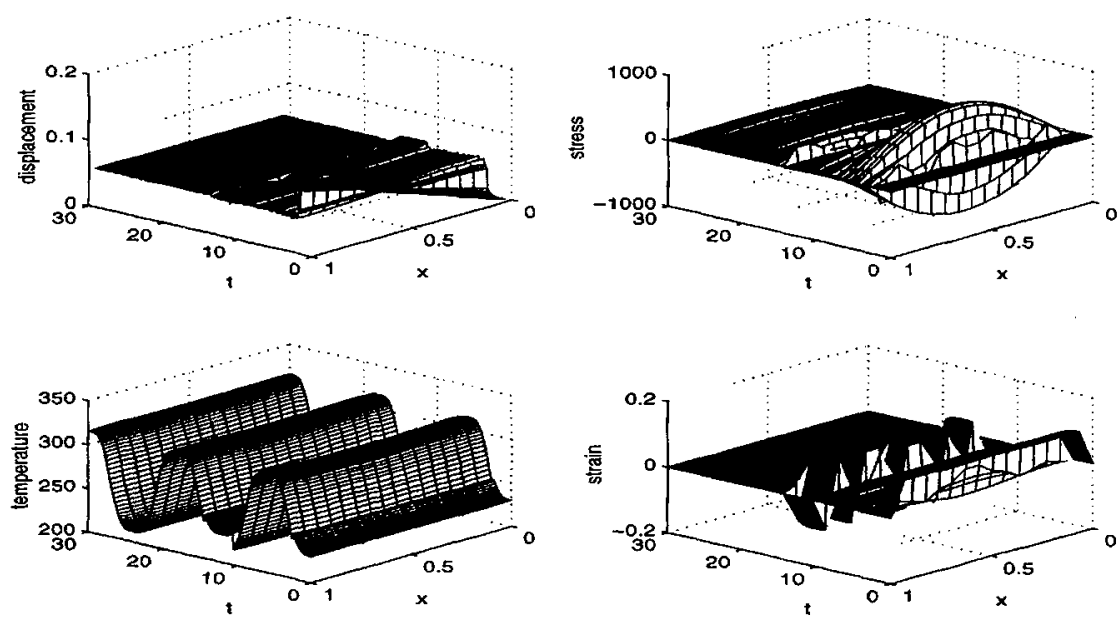

Figure 2: Thermal loading alone might not be able to produce the phase transformation.

where $\rho, k_{i}, i=1,2,3$ are given constants for the alloy $\mathrm{Au}_{23} \mathrm{Cu}_{30} \mathrm{Zn}_{47}$ taken as in [9]. All computations were performed via the reduction of model (9) to a system of differential-algebraic equations and application of an effective DAE solver developed in our previous works [9, 11].

In [9] we showed how a combined boundary stress and thermal control can be used to "guide" the phase transformation in the case when a mechanical loading alone is not sufficient for this purpose. With special and temporal discretisations taken as $0.06 \overline{6}$ and $7.44 \times 10^{-4}$, respectively, in fig. 2 we demonstrate the opposite situation where the thermal loading alone $\left(g=375 \pi \sin ^{3}(\pi t / 6) \mathrm{g} /\left(\mathrm{ms}^{3} \mathrm{~cm}\right)\right)$ is not able to generate the phase transition without additional stress on the boundary of the SMA layer (compare this result with Experiment 2.1 in [9]).

On the other hand, if stress on the boundary is not free, then the assumption of temperature uniformity adopted in [7] may fail as demonstrated by Experiments 1.1, 1.2 in [9]. With spatial and temporal discretisations taken as 0.0625 and $6.7 \times 10^{-4}$, respectively, a typical result of computation with model (9) and boundary interface condition (7) is given in Fig. 3.

In this case the effect of phase transformation slows down the cooling processes of the thermoelectric SMA device.

\section{Discussion and future directions}

Approximations for the interface thermal boundary conditions were derived under simplified assumptions removing which will lead to a much more complex problem for the multilayered SMA structure. One direction of the future development of the presented work is an improved approximation for thermoelectrical fields inside of the semiconductor layers along the lines described in [10] and its coupling to the thermoelectromechanical field of the SMA layer.

In order to increase the frequency of actuation of multilayered SMA structures analysed numerically in this paper, the control by transient response of thermoelectric cooling element should be considered in conjunction with other means of control (in particular, the decrease in 

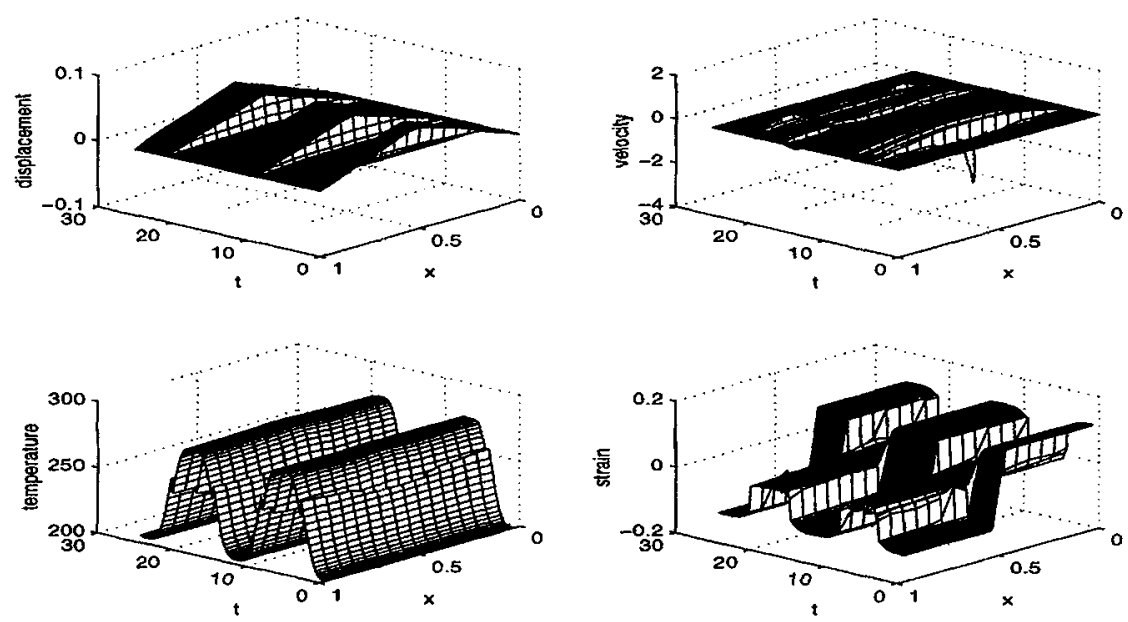

Figure 3: Thermally induced phase transformation in the SMA layer.

hysteresis [9], and the reduction in the thickness of the SMA layer) and with the topological optimisation of the structure.

Acknowledgements Support of CSIRO Mathematical and Information Sciences in providing a travel grant to present this work is gratefully acknowledged.

\section{References}

[1] A. Bhattacharyya et al., Smart Mater. Struct. 4, 252 (1995).

[2] V. Brailovski, F. Trochu, and A. Leboeuf, in Proceedings of the Second International Conference on Shape Memory and Superelastic Technologies, Pacific Grove, California, 1997, edited by A. Pelton et al. (SMST, 1997), p. 227.

[3] Z. Ding and D.C. Lagoudas, SIAM J. Appl. Math. 57, 34 (1997).

[4] P.E. Gray, The Dynamics Behaviour of Thermoelectric Devices (Cambridge Technology Press of the MIT, 1960).

[5] A. F. Ioffe, Semiconductor Thermoelements and Thermoelectric Cooling (Infosearch, London, 1957).

[6] D.C.Lagoudas, Z.Ding, Int.J.Engng.Sci. 33, 2345 (1995).

[7] D.C. Lagoudas, A. Bhattacharyya, Int. J. Solids Structures 35, 331 (1998).

[8] R.V.N. Melnik, Design Autom. Electron. 47124 (1993).

[9] R.V.N. Melnik, A.J.Roberts, K.A.Thomas, Proceedings of SPIE 3667, 290 (1999).

[10] R.V.N. Melnik and H. He, Model. Simul. Mater. Sc. 8, 133 (2000).

[11] R.V.N. Melnik, A.J. Roberts, K.A. Thomas, Comp. Mater. Sci., to appear (2000).

[12] A. R. Shanin et al., J. Intell. Mater. Syst. Struct. 5, 95 (1994). 